\title{
Design and Analysis of Divertor Scraper Elements for the W7-X Stellarator*
}

\author{
J.D. Lore ${ }^{1}$, T. Andreeva ${ }^{2}$, J. Boscary ${ }^{3}$, S. Bozhenkov², J. Geiger ${ }^{2}$, J.H. Harris ${ }^{1}$, H. Hoelbe ${ }^{2}$, A. Lumsdaine ${ }^{1}$, D. \\ McGinnis ${ }^{1}$, A. Peacock ${ }^{3}$, and J. Tipton ${ }^{4}$ \\ ${ }^{1}$ Oak Ridge National Laboratory, Oak Ridge, TN, USA. \\ ${ }^{2}$ Max Planck Institute for Plasma Physics, EURATOM-Association, Greifswald, Germany. \\ ${ }^{3}$ Max Planck Institute for Plasma Physics, EURATOM-Association, Garching, Germany. \\ ${ }^{4}$ University of Evansville, Evansville, IN, USA. \\ Corresponding author e-mail: lorejd@ornl.gov.
}

\begin{abstract}
A set of new water-cooled divertor components is being designed for the Wendelstein 7-X (W7-X) stellarator to protect the edges of the primary plasma facing components during the bootstrap current evolution ( 40s). These new components, referred to as 'scraper elements', will intercept field lines and associated heat flux that would otherwise overload the main target edges in certain operational scenarios. The scraper elements are calculated to experience peak heat fluxes $\sim 15$ $16 \mathrm{MW} / \mathrm{m}^{2}$ and will be constructed from carbon fiber reinforced composite monoblocks of a type that has been qualified for ITER. The heat flux distribution and magnitude is calculated from field line following in a 3D magnetic field that includes the contribution from plasma currents. The heat flux calculations are coupled with an engineering design in an iterative process to generate scraper elements that meet the design criteria while reducing the geometric complexity of the elements.
\end{abstract}

Keywords- Wendelstein 7-X; Stellarator; Divertor; Heat flux; Island Divertor

\section{INTRODUCTION}

The issue of power and particle exhaust, particularly during steady-state operation, is common to tokamaks and stellarators. The ITER divertor, for example, is expected to experience heat fluxes on the order of $10 \mathrm{MW} / \mathrm{m}^{2}$ for hundreds of seconds [1]. Demonstrating the feasibility of a steady-state, high-heat-flux (HHF) divertor configuration with actively-cooled plasma facing components (PFCs) is one of the design goals for the Wendelstein 7-X (W7-X) stellarator. W7-X, is a large stellarator $\quad(\langle\mathrm{R}\rangle=5.5 \mathrm{~m}, \quad\langle\mathrm{a}\rangle=0.53 \mathrm{~m})$ currently under construction in Greifswald, Germany, with initial plasma operations scheduled for 2015.

W7-X will operate in three main phases. In the first phase (OP1.1), commissioning plasmas will be run with a reduced set of in-vessel components. For the next phase, (OP1.2) an inertially cooled divertor will be installed, PFCs will be mostly un-cooled, and the input power and pulse length will be limited. For the third phase (OP2), HHF water-cooled divertor components will be installed, and high-power, long pulse (up to 30 minutes) operation is planned. The HHF divertor consists of 10 nearly identical units, one of which is shown in Fig. 1. The vertical and horizontal targets, as well as the 'high iota tail', comprise the primary components, and are rated for $10 \mathrm{MW} / \mathrm{m}^{2}$ over the main operational area. However, as a result of the cooling pipe geometry, the target elements near the pumping gap are limited to $5 \mathrm{MW} / \mathrm{m}^{2}$ [2]. Transport simulations of certain experimental scenarios expected to have good particle confinement predict an overload of the target edges. There are several potential solutions to this problem, including using the auxiliary coil system to reposition the plasma, changing the plasma boundary shape using electron cyclotron current drive, and avoiding high-power operation in the problematic scenarios. This paper does not discuss the merits of each possible method, but instead presents one solution involving an additional plasma facing component that will protect the overloaded areas by intercepting the incident flux. The scraper element (SE) [3] will be constructed from watercooled carbon fiber reinforced composite (CFC) monoblocks, which are rated for $20 \mathrm{MW} / \mathrm{m}^{2}$. The present $\mathrm{W} 7-\mathrm{X}$ planning allows for the installation of 1-2 adiabatic SEs during OP1.2. A

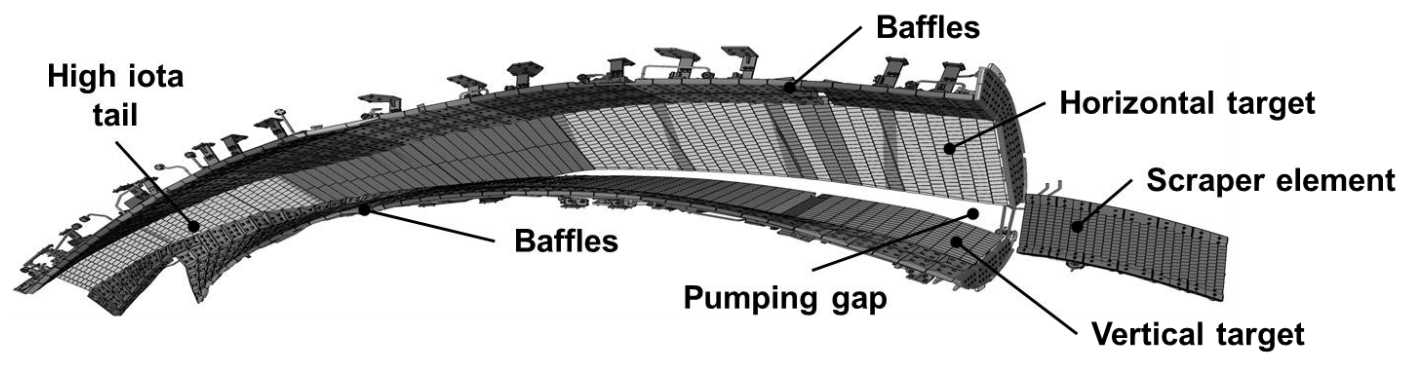

Fig. 1. The components of the divertor unit for W7-X.

*This work is supported by the US Department of Energy, Contract DE-AC05-00OR22725. 

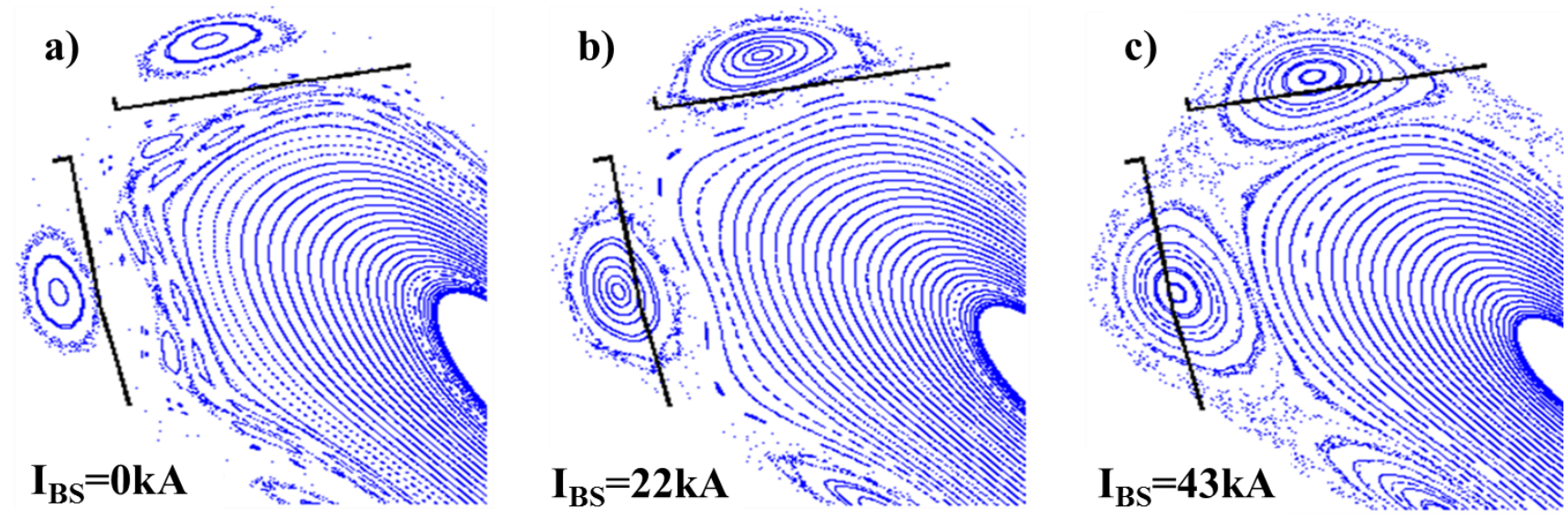

Fig. 2. Effect of the bootstrap current evolution on the plasma shape when the vacuum rotational transform has been reduced below 1 . Target plates are shown as black lines.

full set of 10 water-cooled SEs would potentially be installed in OP2. In this paper we describe the design process and current status of the scraper element, with a focus on the plasma physics considerations. Heat transfer and cooling systems properties are presented in [4].

\section{PREDICTED OVERLOAD OF COMPONENTS DURING BOOTSTRAP CURRENT EVOLUTION}

In the 'standard' W7-X magnetic configuration, where all primary coils are energized with identical current magnitude, the boundary of the confinement region is defined by the separatrix of a $5 / 5$ island chain intersected by target plates. The 'island divertor' (ID) concept was successfully tested on Wendelstein 7-AS [5] and on LHD as a 'local island divertor' [6]. Fig. 2c shows an example of a W7-X ID configuration. An ID can be thought of as an extension of a tokamak poloidal divertor [7], however, the x-points have a helical pitch corresponding to the value of the rotational transform at the boundary $\left(l_{\mathrm{B}}\right)$, and the magnetic field and PFCs are not toroidally symmetric. Throughout the discharge, $l_{B}$ must be held approximately constant to maintain the position of the separatrix relative to the target plates, and acceptable particle and heat flux deposition patterns and magnitudes.

One of the optimization targets for $\mathrm{W} 7-\mathrm{X}$ was a small bootstrap current. Deviation from the 'minimum bootstrap current' configuration which resulted from the optimization procedure generally leads to a finite bootstrap current that evolves on the L/R timescale, ( 40s) [8]. While the bootstrap current is still small relative to a comparable tokamak, as the bootstrap current increases, the rotational transform profile evolves, which can result in changes to $l_{\mathrm{B}}$ on the order of $10 \%$. If the plasma configuration has an ID shape in vacuum, an increasing bootstrap current tends to increase $l_{B}$, moving the $5 / 5$ island chain inward in minor radius, reducing the volume of the confinement region and eventually resulting in a non-ID shape. In the standard configuration a steady-state bootstrap current of $\mathrm{I}_{\mathrm{BS}}=43 \mathrm{kA}$ has been calculated by transport simulations [8]. The final plasma equilibrium resulting has a limited shape and proper ID operation cannot be expected.

To account for this change the vacuum transform can be reduced such that the initial configuration is limited by the horizontal divertor plate (Fig. 2a) and an ID shape with $\mathrm{l}_{\mathrm{B}}=1$ is achieved when the bootstrap current is fully evolved (Fig. 2c). The calculated magnetic field line strike patterns onto the horizontal and vertical targets during the bootstrap current evolution are shown in Fig. 3a-c. At $\mathrm{I}_{\mathrm{BS}}=0 \mathrm{kA}$ the limited shape results in a highly localized pattern on the horizontal plate, while at $\mathrm{I}_{\mathrm{BS}}=43 \mathrm{kA}$ the toroidal 'stripes' associated with the ID shape are restored. (Note that a limited shape is acceptable

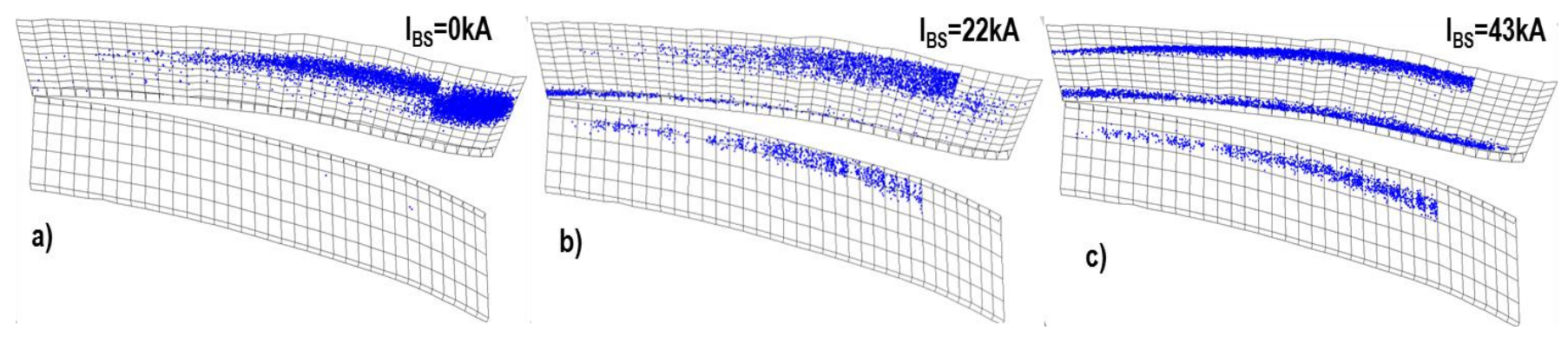

Fig. 3. Magnetic field line strike point patterns on the horizontal and vertical targets during the bootstrap current evolution. 


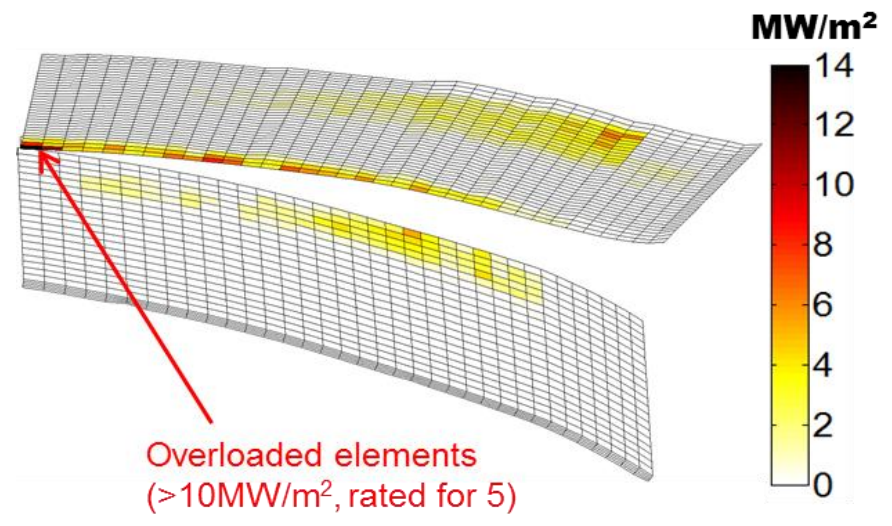

Fig. 4. Heat fluxes incident on the horizontal and vertical target plates with no scraper element at $\mathrm{I}_{\mathrm{BS}}=22 \mathrm{kA}$.

early in the discharge when the input power is low).

While this addresses the $\mathrm{I}_{\mathrm{BS}}=43 \mathrm{kA}$ case, as the configuration evolves the heat flux patterns sweep across edges of the main target plates causing overload. The intermediate value of $\mathrm{I}_{\mathrm{BS}}=22 \mathrm{kA}$ has been identified as a 'worst case', with the edge of the horizontal target calculated to receive $>10 \mathrm{MW} / \mathrm{m}^{2}$ (rated for $5 \mathrm{MW} / \mathrm{m}^{2}$ ) and baffle components rated for $0.5 \mathrm{MW} / \mathrm{m}^{2}$ near the pumping gap $>30 \mathrm{MW} / \mathrm{m}^{2}$. Fig. 4 shows the calculated heat fluxes to the horizontal and vertical target plates at $\mathrm{I}_{\mathrm{BS}}=22 \mathrm{kA}$. Although these loads are transient on the magnetic equilibrium evolution timescale, they are effectively steady-state with respect to the thermal properties of the PFCs and cooling system and must be mitigated. The SE has been proposed to protect the target edges during the bootstrap current evolution by intercepting magnetic field lines and associated plasma fluxes incident on the overloaded areas. The

a)
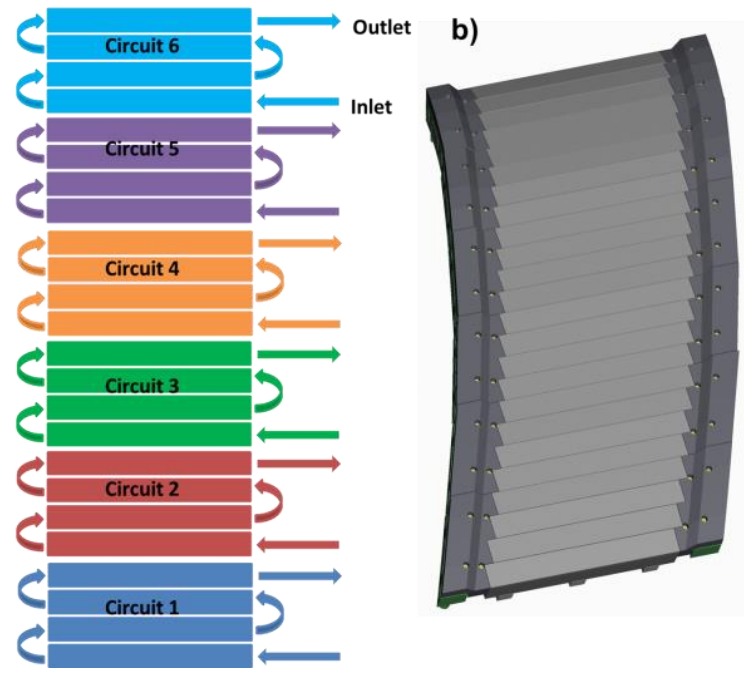

Fig. 5. Arrangement of the SE cooling circuits (a) and current SE design (b)
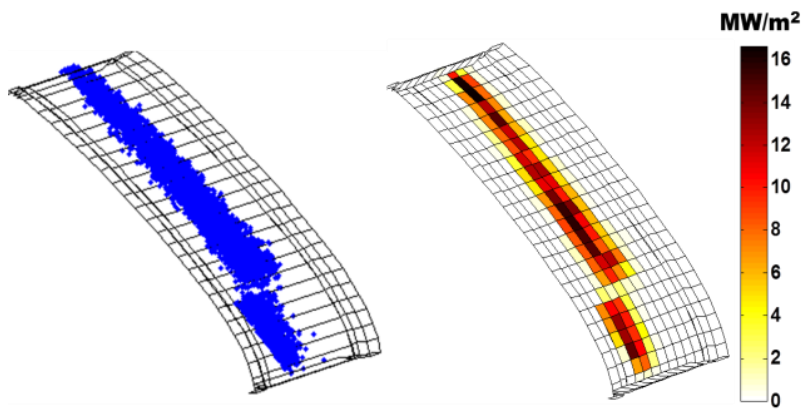

Fig. 6. (left) Strike point pattern and (right) convective heat fluxes on the $\mathrm{SE}$ in the $22 \mathrm{kA}$ magnetic configuration.

design of the SE is discussed in the following section.

\section{DESIGN OF THE SCRAPER ELEMENT}

The current SE design consists of 24 CFC monoblock fingers arranged in 6 poloidal circuits, as shown in Fig. 5. The monoblock fingers are $2.7 \mathrm{~cm}$ wide by $24.7 \mathrm{~cm}$ long, and the SE has a plasma-facing surface area of $\sim 0.16 \mathrm{~m}^{2}$. (A 32 monoblock finger SE was previously proposed [9]; the current SE has been shortened to reduce cost, at the expense of higher average and peak heat fluxes.) One $\mathrm{SE}$ is required to protect each of the 10 divertor modules.

The SE shape and position is designed considering constraints due to plasma physics (heat fluxes, pumping efficiency, magnetic field models), fluid dynamics (cooling water pressure drop and temperature rise), and engineering (manufacturing and alignment tolerances, available space). The initial design process consisted of iteration between heat flux calculations using the DIV3D code [9], and CAD manipulation. At each iteration DIV3D reads in the CAD model, calculates the heat flux to the plasma facing surfaces and, and a new optimal surface is generated considering constraints on the shape and geometry of the CFC monoblocks. Accounting for engineering constraints avoids 'ideal' surfaces from a physics perspective that are prohibitively difficult to design and construct. For example, 'poloidal' curvature (in line with the cooling pipe) of the monoblocks is minimized due to limitations of the CFC fabrication procedure. The new surface generated by DIV3D is then loaded into a flexible CAD model that best matches the surface using the actual monoblock geometry.

DIV3D calculates the heat fluxes incident on arbitrary plasma facing surfaces in 3D magnetic fields. For these calculations, the magnetic field model combines contributions from the nested flux surface equilibrium calculated by the nonlinear ideal magnetohydrodynamics code VMEC [10,11] with the field generated by the external coils using a 'virtual casing' principle calculated using the EXTENDER code [12]. Magnetic field errors due to coil misalignment are not included. Error fields can completely change the structure of the heat flux, for example a resonant error field can result in a plasma boundary shape that causes nearly all of the input power to be deposited on in a single half field period (a 10x increase) [13]. Effects that cause a large deviation from the 


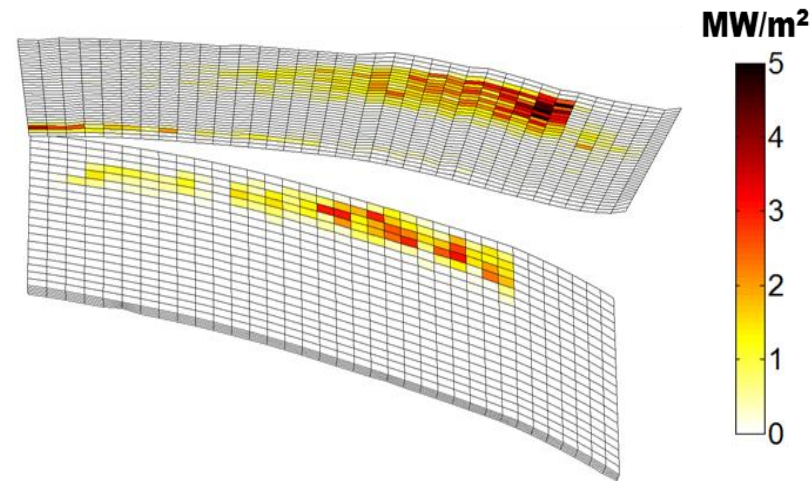

Fig. 7. Heat flux incident on the horizontal and vertical targets with the $\mathrm{SE}$ in place at $\mathrm{I}_{\mathrm{BS}}=22 \mathrm{kA}$.

device symmetry are unacceptable and minimization is a priority during device construction and alignment. In addition, error fields will be minimized with a set of trim coils. The effect of the total field on the heat flux calculations presented here will be determined when final coil alignment is completed. It is assumed that the heat transport is described by free-streaming parallel convection with random-walk perpendicular diffusion. This model is implemented by initiating a large number of field lines along a field line that traces out a flux surface near the magnetic separatrix The field lines are traced in both directions, with randomly directed perpendicular steps of size $\Delta_{\perp}$ introduced corresponding to a magnetic diffusivity $\mathrm{D}_{\mathrm{m}} \sim\left(\Delta_{\perp}\right)^{2} / \Delta_{\|}$, where $\Delta_{\|}$is the step size parallel to the magnetic field used when integrating the field line differential equation. The magnetic diffusivity is a free parameter related to plasma transport quantities as $D_{m}=\chi_{\perp} / v_{T}$, where $\chi_{\perp}$ is the thermal diffusivity and $v_{T}$ the thermal velocity. The lines are traced until an intersection with a plasma facing component is found.

To convert the resulting strike patterns into a heat flux the total input power (10MW) is assumed to cross the last closed flux surface uniformly, with an equal fraction of the power

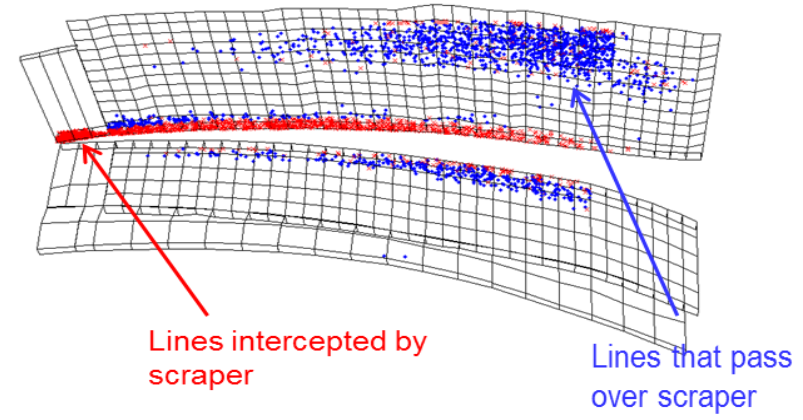

Fig. 8. Strike patterns on the horizontal and vertical target plates and associated baffles with the $\mathrm{SE}$ in place at $\mathrm{I}_{\mathrm{BS}}=22 \mathrm{kA}$. Blue points are field lines that pass over the SE, red points indicate field lines that would intersect the plotted componentes if the SE were removed. assigned to each initial point. The PFC surfaces are then subdivided into a series of area 'bins', and the total power carried to each bin is divided by the area to calculate the heat flux. This is illustrated in Fig. 6, where each blue dot in Fig. 6a represents a field line intersection, and the power density incident on the element is shown in Fig. 6b. For the simulations presented here $D_{m}=3.2 \cdot 10^{6} \mathrm{~m}^{2} / \mathrm{m}$ was chosen to match previous heat load calculations for other W7-X components [9]. A typical computation with 10000 launch point with this value of $\mathrm{D}_{\mathrm{m}}$ including all of the PFCs shown in Fig. 1 takes on the order of three hours. Generally, increasing $\mathrm{D}_{\mathrm{m}}$ will result in broader flux patterns (width $\sim D_{m}{ }^{1 / 2}$ ) and a decreased peak heat flux $\left(Q_{\max } \sim D_{m}{ }^{-1 / 2}\right)$. The robustness of the presented results with respect to $D_{m}$ will be evaluated as part of the sensitivity study to be performed for the final SE design, however the same transport assumptions were used to design the entire target module so the SE is not taking additional risk with respect to the other plasma facing components. Currently 1-2 inertially cooled SE are being considered for the end of OP1. Infrared camera measurements will be used to measure the heat flux magnitude and patterns, and these results can be used to 'calibrate' the transport model.

Radiation is not included in the surface power load or as an energy sink in the plasma transport. The resulting power loads are therefore expected to be conservative as line radiation and charge exchange radiation are expected to reduce the energy directly deposited to the strike locations (the radiated power must of course reach a PFC, however it will be spread over a larger surface area). In addition, at lower plasma collisionality it is expected that the edge plasma will develop parallel temperature gradients, reducing the electron temperature at the target and therefore the heat flux. To account for these effects, a more complete transport calculation must be performed, for example using the EMC3-EIRENE code [14,15]. The additional computational requirements, however, make an iterative design procedure prohibitive.

The SE shown in Fig. 5 was produced using the above method, using the $22 \mathrm{kA}$ magnetic configuration as a design point. The lighter gray area in Fig. 5b is CFC (monoblocks), and the darker areas along the edges are graphite tiles that

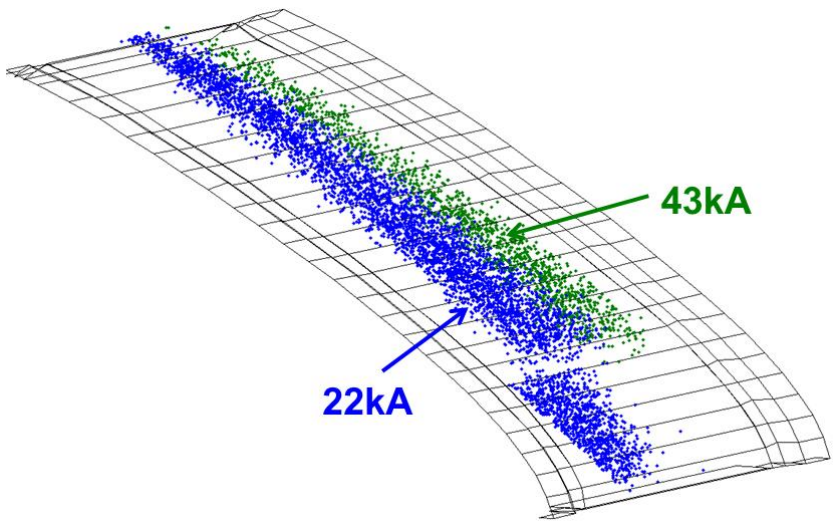

Fig. 9. Strike patterns on the $\mathrm{SE}$ at $\mathrm{I}_{\mathrm{BS}}=22 \mathrm{kA}$ (blue) and $\mathrm{I}_{\mathrm{BS}}=43 \mathrm{kA}$ (green). 

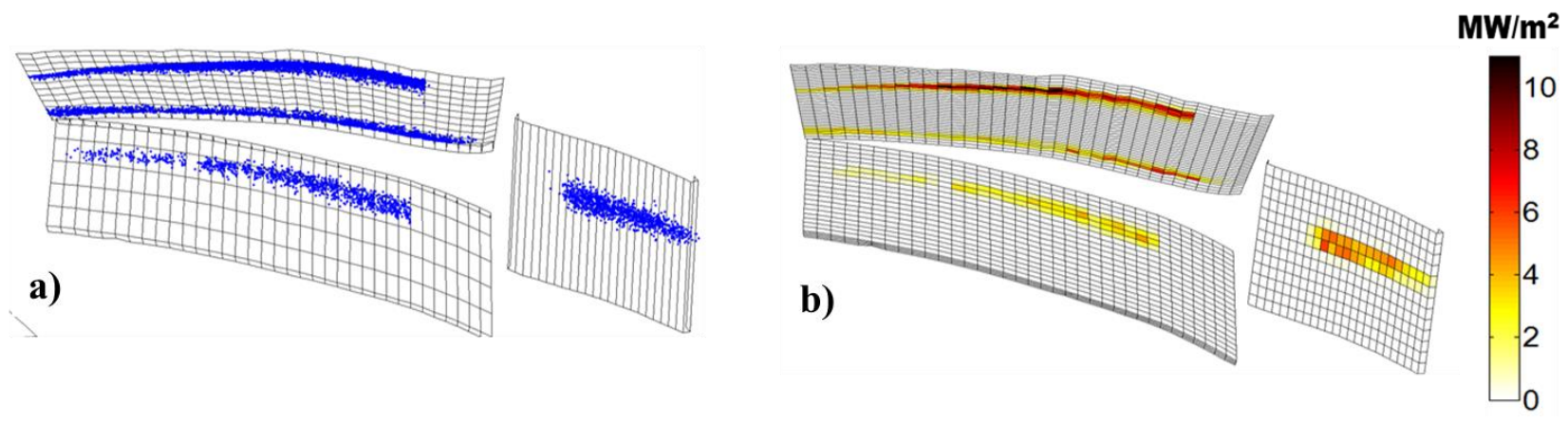

Fig. 10. Strike patterns (a) and heat fluxes (b) to the target plates and $S E$ at $I_{B S}=43 k A$.

protect the cooling water pipes. The calculated heat fluxes and strike point pattern is shown in Fig. 6. The peak value of $\sim 16 \mathrm{MW} / \mathrm{m}^{2}$ is below the rated value of $20 \mathrm{MW} / \mathrm{m}^{2}$, with a statistical uncertainty in the heat flux magnitude on the order of $10 \%$.

Fig. 7 shows the heat fluxes on the horizontal and vertical target plates with the SE in place. The heat flux on the lower rated components along the target edges has been almost completely removed (last row of rectangles in Fig. 7). The peak heat flux has also been reduced, with the maximum below $5 \mathrm{MW} / \mathrm{m}^{2}$. Fig. 8 shows directly the effect of the SE on the strike patterns to the main target plates and associated baffle components. The blue points indicate field lines that intersect the plotted components with the SE in place, i.e., field lines that pass over the SE. The red points are lines that would carry heat to the components if the SE were removed, but are now intercepted. As shown, most of the lines that are intercepted are along the target edges, where the components would otherwise be overloaded. At $22 \mathrm{kA}$ the SE receives approximately $55 \%$ of the total input power.

\section{EVALUATION OF THE SCRAPER ELEMENT AT $\mathrm{I}_{\mathrm{BS}}=43 \mathrm{KA}$}

As discussed above, the primary purpose of the SE is to protect the areas of the divertor components that are overloaded during the bootstrap current evolution, with $\mathrm{I}_{\mathrm{BS}}=22 \mathrm{kA}$ identified as a worst case scenario and used as the design point for the SE. As the SE was not part of the divertor module as originally designed, it would ideally be unloaded after the current evolution was completed. Fig. 10 shows the strike patterns and heat fluxes to the target plates and SE at $\mathrm{I}_{\mathrm{BS}}=43 \mathrm{kA}$. The SE intercepts a smaller percentage of the total input power, $\sim 14 \%$, with a peak flux to the SE of $\sim 7 \mathrm{MW} / \mathrm{m}^{2}$. Given the space limitations and the geometric constraints imposed, it was not possible to completely unload the SE at $43 \mathrm{kA}$. The reason for this is that the plasma shape does not dramatically change as the current is increased from $22 \mathrm{kA}$ to $43 \mathrm{kA}$. The flux tubes that carry the majority of the convective load to the target edges [9], i.e., the load to be intercepted at $22 \mathrm{kA}$, shifts only a few $\mathrm{cm}$ poloidally. While this shift is sufficient to move the strike patterns on the horizontal target to the lower rated area, the intersection of the flux tube with the SE primarily moves in-line with the surface, see Fig. 9. In principle this intersection could be avoided by introducing a strong poloidal curvature to the SE, however this causes interference with the vacuum vessel, in addition to complicating the monoblock manufacturing process. At both $22 \mathrm{kA}$ and $43 \mathrm{kA}$ the graphite protecting the piping does not receive a convective heat flux, and is expected to only carry radiative loads.

The interception of field lines and heat by the SE at $43 \mathrm{kA}$ also has implications for the pumping properties of W7-X. Most of the flux removed at 43kA is from along the edge of the horizontal target near the pumping gap, as in the $22 \mathrm{kA}$ case. The modification to the strike pattern is shown in Fig. 11. This has the effect of reducing the neutral pressure in the pumping volume, as neutral particles produced by surface recombination and re-emission have a higher probability of entering the pumping region if the incident ions are near the pump entrance [16]. The impact of the SE on the pumping efficiency and neutral particle transport is currently being investigated using the 3D scrape-off-layer transport code EMC3-EIRENE $[14,15]$.

\section{SUMMARY AND ONGOING WORK}

The scraper element is designed to protect overloaded areas of the primary divertor module during the bootstrap current evolution in certain magnetic configurations. Without the SE,

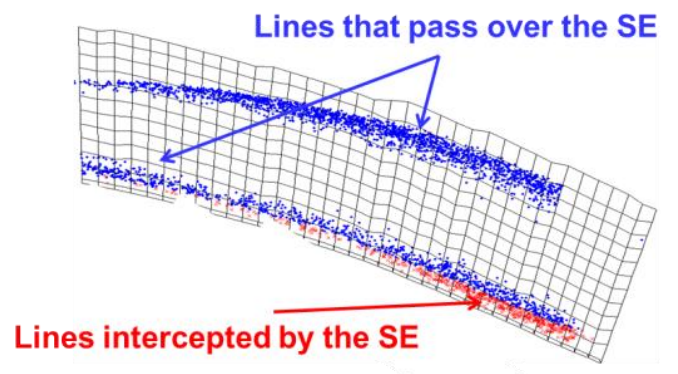

Fig. 11. Strike patterns on the horizontal target plate with the SE in place at $I_{B S}=43 k A$. Blue points are field lines that pass over the $\mathrm{SE}$, red points indicate field lines that would intersect the plotted componentes if the SE were removed. 
regions of the horizontal target rated for $5 \mathrm{MW} / \mathrm{m}^{2}$ are expected to receive $>10 \mathrm{MW} / \mathrm{m}^{2}$. Using a design point of $\mathrm{I}_{\mathrm{BS}}=22 \mathrm{kA}$, the SE surface was designed by iterating heat flux calculations with CAD manipulations, incorporating constrains from both plasma physics and engineering considerations. The current SE design reduces the primary target and baffle loads to acceptable levels, and a peak SE flux of $\sim 16 \mathrm{MW} / \mathrm{m}^{2}$ is calculated. This load is significantly reduced at the fully-evolved bootstrap current value of $43 \mathrm{kA}$, however some flux that would be incident on the target edges is intercepted. This is expected to reduce the neutral pressure in the pumping volume, and is a current area of investigation. The effect of the SE has also been assessed in other magnetic configurations, with no interference with standard operation found. A sensitivity analysis to uncertainties in the plasma transport model and the engineering and installation procedures is currently in progress.

\section{ACKNOWLEGEMENT}

This manuscript has been authored by UT-Battelle, LLC, under Contract No. DE-AC05-00OR22725 with the U.S. Department of Energy. The United States Government retains and the publisher, by accepting the article for publication, acknowledges that the United States Government retains a non-exclusive, paid-up, irrevocable, world-wide license to publish or reproduce the published form of this manuscript, or allow others to do so, for United States Government purposes.

\section{REFERENCES}

[1] A. Loarte, B. Lipschultz, A.S. Kukushkin, G.F. Matthews, P.C. Stangeby, N. Asakura, et al., "Chapter 4: Power and particle control," Nucl. Fusion, Vol. 47 (2007) S303.

[2] J. Boscary, A. Peacock, T. Friedrich, H. Greuner, B. Boesworth, H. Tittes, et al., "Design improvement of the target elements of Wendelstein 7-X divertor", Fus. Eng. Des., Vol. 87 (2012) 1453.
[3] A. Peacock, J. Kisslinger, J. Boscary, J. Geiger, F. Hurd, R. Koenig, et al., "A proposed scraper element to protect the end of the W7-X divertor target elements", IEEE/NPSS 24th Symposium on Fusion Engineering, (2011) SP2-41.

[4] A. Lumsdaine, et al., These Proceedings.

[5] P. Grigull, K. McCormick, J. Baldzuhn, R. Burhenn, R. Brakel, H. Ehmler, et al., "First island divertor experiments on the W7-AS stellarator", Plasma Phys. Control. Fusion Vol. 43 (2001) A175.

[6] T. Morisaki, S. Masuzaki, H. Suzuki, A. Komori, N. Ohyabu, O. Motojima, et al., "Local Island Divertor Experiment", Fusion Sci. Technol. Vol. 58 (2010), No 1, 232-241.

[7] Y. Feng, F. Sardei, P. Grigull, K. McCormick, J. Kisslinger, D. Reiter, "Physics of island divertors as highlighted by the example of W7-AS", Nucl. Fusion Vol. 46 (2006) 807

[8] J. Geiger, C.D. Beidler, M. Drevlak, H. Maassberg, C. Nuehrenberg, Y. Suzuki, et al., "Effects of net currents on the magnetic configuration of W7-X", Contrib. Plasma Phys. Vol. 50 (2010) 770.

[9] J. Lore, T. Andreeva, J. Boscary, J.M. Canik, J. Geiger, J.H. Harris, et al., "Heat flux and design calculations for the W7-X divertor scraper element," Proceedings of the $24^{\text {th }}$ International Atomic Energy Agency Fusion Energy Conference, (2012), FTP/P1-02.

[10] S.P. Hirshman and J.C. Whitson, "Steepest-descent moment method for three-dimensional magnetohydrodynamic equilibria," Phys. Fluids, Vol. 26, (1983), 3553.

[11] S.P. Hirshman and W.I. van Rij, "Three-dimensional free boundary calculations using a spectral Green's function method", Comp. Phys. Commun., Vol. 43 (1986) 143.

[12] M. Drevlak, D. Monticello and A. Reiman, "PIES free boundary stellarator equilibria with improved initial conditions", Nucl. Fusion, Vol. 45 (2005), 731.

[13] J. Kisslinger, private commuication.

[14] Y. Feng, F. Sardei, J. Kisslinger, and P. Grigull, "A 3D monte carlo code for plasma transport in island divertors,", J. Nucl. Mater, Vol. 241243 (1997), 930.

[15] Y. Feng, F. Sardei, and J. Kisslinger, "3D fluid modeling of the edge plasma by means of a Monte Carlo technique", J. Nucl. Mater., Vol. 266-269 (1999), 812.

[16] R. Maingi, J.G. Watkins, M.A. Mahdavi, L.W. Owen, "Pump plenum pressure dependence on divertor plasma parameters and magnetic geometry in the DIII-D tokamak," Nucl. Fusion, Vol. 39, (1999) 1187. 